Annals of the Rheumatic Diseases, 1989; 48, 163-165

Case report

\title{
Chronic synovitis of the shoulder in familial Mediterranean fever: a disease of symptoms not signs
}

\author{
R A HUGHES AND J T SCOTT \\ From the Department of Rheumatology, Charing Cross Hospital, London
}

SUMMARY Severe shoulder pain in a 41 year old Arab woman persisted for three months despite non-steroidal anti-inflammatory drug treatment. Isotope bone scanning and computed tomography showed inflammation of the glenohumeral joint and a large effusion, which needle aspiration had initially failed to reveal. A diagnosis of familial Mediterranean fever was made on the basis of a strongly suggestive past personal and family history, sterility of the joint effusion, and a good response to colchicine.

Key words: arthritis, colchicine.

Familial Mediterranean fever is a disease which is commonly encountered in ethnic groups of Mediterranean origin. It is an autosomal recessive condition, which is manifest as episodic fevers, pleuritis, peritonitis, and arthritis; the aetiology is unknown. No definitive diagnostic test exists; episodes are usually short in duration and self limiting, and there is a high incidence of secondary amyloidosis in chronic cases.

The arthritis of familial Mediterranean fever has been extensively documented in a series of large reviews. $^{1-4}$

Joint involvement occurs either in an acute form lasting only a few days with complete resolution of symptoms, usually as a monarthritis of the lower limb or, alternatively, as a more chronic disease lasting many months often with resulting joint damage.

The purpose of reporting this case is threefold. Firstly, with a large population of people of Mediterranean origin living in our community and an equally sizable group seeking treatment from abroad attention should be drawn to a condition which has been infrequently encountered by practising rheumatologists in this country. Secondly, it

Accepted for publication 13 June 1988

Correspondence to Dr R A Hughes, Department of Rheumatology, Charing Cross Hospital (Fulham), Fulham Palace Road, London W6 8RF. demonstrates how non-invasive methods of visualising the joint have an important part to play in the investigation of a condition where the extent of inflammation may not be apparent clinically and, finally, colchicine is shown, in this case, to be effective in achieving resolution of a refractory synovitis in familial Mediterranean fever despite previous reports to the contrary. ${ }^{1}$

\section{Case report}

A 41 year old Arab woman living in Egypt presented in March 1987 to the Maadi Hospital, Cairo, with a two month history of increasing pain associated with any movement of her right shoulder. She also complained of an irregular fever.

In Cairo she was treated with intramuscular streptomycin and oral tetracycline for brucellosis, which was thought to account for her symptoms. She did not improve on this regimen. A wide variety of non-steroidal anti-inflammatory drugs also made no difference to the pain.

She was seen in London one month later. Specific questioning uncovered a long history of short lived episodes of upper abdominal pain and fever over the preceding 10 years, to which the patient had attached little significance. She could, however, remember being hospitalised at the age of 9 with a swollen right knee, and again at the age of 14 with a 
febrile episode in conjunction with a further inflammation of the same knee. Her younger sister of 38 apparently had a similar past medical history suggestive of familial Mediterranean fever.

On examination there was a striking discrepancy between the now severe pain related to the right shoulder and the lack of outward signs of inflammation. Active and passive movement of her right arm was precluded by intense pain experienced in the shoulder, upper arm, and axillary regions. No local tenderness was elicited. Three enlarged tender lymph nodes, however, were palpable in the right supraclavicular fossa. There was a fever of $39 \cdot 5^{\circ} \mathrm{C}$.

Preliminary investigations showed a normal shoulder $x$ ray, a raised erythrocyte sedimentation rate of $73 \mathrm{~mm} / \mathrm{h}$, and $C$ reactive protein raised at 47 units, with a normal total white cell count, haemoglobin, and haemoglobin electrophoresis. Rheumatoid factor, antinuclear antibody, and brucella serology were all negative.

Blood and urine cultures were sterile, and urine analysis showed no proteinuria. The right glenohumeral joint was aspirated by anterior approach and only $2 \mathrm{ml}$ of clear synovial fluid was obtained. Intra-articular steroids were not administered owing to the possibility of local joint sepsis as a diagnosis. No crystals were seen and cell count showed a moderate neutrophilia. The fluid was sterile.

Isotope bone scanning showed a hot right shoulder (Fig. 1) and computed tomography (CT) scanning of this area allowed visualisation of a large previously unsuspected joint effusion (Fig. 2).

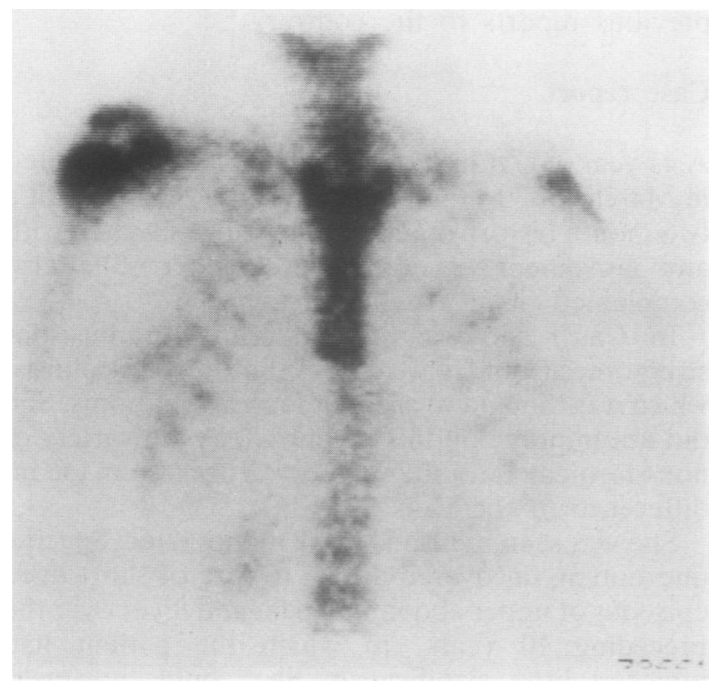

Fig. 1 Technetium bone scan showing increased uptake of isotope in association with the right shoulder joint.

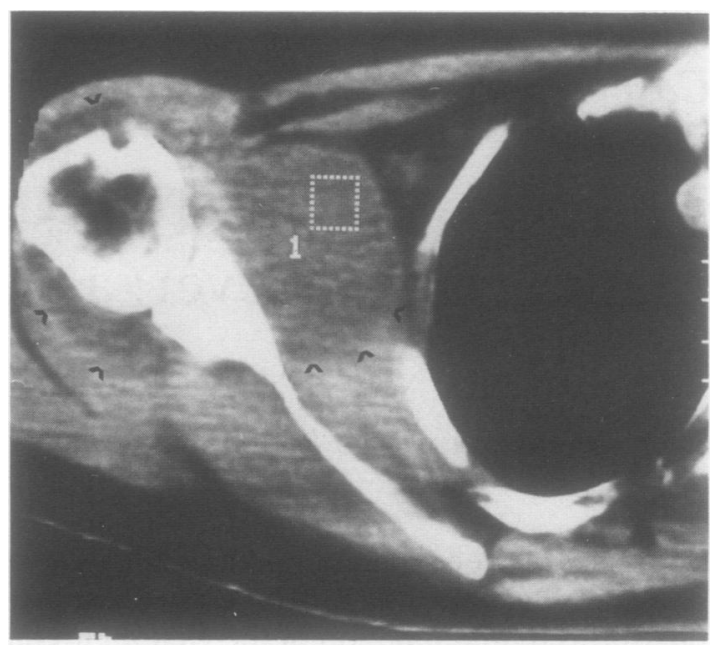

Fig. 2 Computed tomography scan of the right shoulder. Areas of the same density as that contained in square 1 represent synovial effusion. The extent of effusion is indicated by the arrows.

The patient was treated with $1.5 \mathrm{mg}$ oral colchicine daily and both her shoulder pain and her fever settled rapidly. Three days later she was discharged from hospital, asymptomatic, with a shoulder both clinically and radiologically normal. No repeat erythrocyte sedimentation rate, $\mathrm{C}$ reactive protein, or CT scan were obtained. Local lymphadenopathy was still present on discharge, though tenderness was reduced. She has been advised to continue with prophylactic colchicine treatment. No further problems have been reported over the ensuing one year period.

\section{Discussion}

Arthritis may occur in up to $70 \%$ of cases of familial Mediterranean fever. ${ }^{135}$ Onset is usually under the age of 15 , and initial diagnosis may be made more difficult by the occurrence of joint symptoms as the major presenting complaint, often in the absence of accompanying fever or polyserositis. This has often led to misdiagnosis and inappropriate treatment. The intensity of the shoulder pain and the associated loss of limb function were remarkable in this case.

Although the joints of the lower limb are most commonly affected, arthritis has been reported to occur at a wide variety of other sites, including the shoulder, the cervical spine ${ }^{6}$ and the temporomandibular joint. ${ }^{7}$ 
As with the hip joint assessment of the degree of inflammation can be made difficult by the overlying musculature ensheathing the joint. CT scanning has added a new dimension to the non-invasive visualisation of these and other joints, as evident in this case. The extent of the effusion was apparent only from the scanning image. It is possible that joint fluid was not aspirated owing to its location in the subscapular bursa as shown on the CT image. A conventional anterior approach was performed.

Although in this patient no residual joint damage occurred, there are now many reports of chronic familial Mediterranean fever arthritis subsequently leading to both premature osteoarthritic changes and avascular necrosis. ${ }^{589}$ This occurs especially with hip involvement and is thought to relate to associated vascular insufficiency. There has been no evidence of amyloid deposition in such hip joints and histology has shown only non-specific synovial inflammation.

It has been well established that colchicine taken on a regular long term basis is an effective prophylactic against recurrent attacks of familial Mediterranean fever. ${ }^{10-12}$ Its efficacy in the treatment of synovitis has previously been doubted, however. $^{310} 12$ The present case indicates that the drug should at least be tried as initial treatment in the established attack. There is little evidence to suggest that local intra-articular steroid injection is of any benefit in such a synovitis.

\section{References}

1 Heller H, Gafni J, Michaeli D, et al. The arthritis of familial Mediterranean fever. Arthritis Rheum 1966; 9: 1-17.

2 Schwabe A D, Peters R B. Familial Mediterranean fever in Armenians. Analysis of 100 cases. Medicine (Baltimore) 1974; 53: 453-62.

3 Sohar E, Gafni J, Pras M, Heller H. Familial Mediterranean fever. A survey of $\mathbf{4 7 0}$ cases and review of the literature. Am J Med 1967; 43: 227-53.

4 Makin M, Levin S. The articular manifestations of periodic disease (familial Mediterranean fever). J Bone Joint Surg [Am] 1965; 47: 1615-9.

5 Sneh E, Pras M, Michaeli D, Shahin N, Gafni J. Protracted arthritis in familial Mediterranean fever. Rheumatology and Rehabilitation 1977; 16: 102-6.

6 Sukenik S, Horowitz J, Boehm R, Bar-Ziv J. Cervical spine involvement in familial Mediterranean fever. J Rheumatol 1985; 12: $603-4$.

7 Cooksey D E, Girard K. Temporomandibular joint synovitis with effusion in familial Mediterranean fever. Oral Surg Oral Med Oral Pathol 1979; 47: 123-6.

8 Herness D, Makin M. Articular damage in familial Mediterranean fever. Report of four cases. J Bone Joint Surg [Am] 1975; 57: 265-7.

9 Kaushansky K, Finerman GAM, Schwabe AD. Chronic destructive arthritis in familial Mediterranean fever: the predominance of hip involvement and its management. Clin Orthop 1981; 155: 156-61.

10 Zemer D, Revach M, Pras M, et al. A controlled trial of colchicine in preventing attacks of familial Mediterranean fever. N Engl J Med 1974; 291: 932-4.

11 Goldstein R C, Schwabe A D. Prophylactic colchicine therapy in familial Mediterranean fever. A controlled, double-blind study. Ann Intern Med 1974; 81: 792-4.

12 Dinarello C A, Wolff S M, Goldfinger S E, Dale D C, Alling D W. Colchinine therapy for familial Mediterranean fever. A double-blind trial. $N$ Engl J Med 1974; 291: 934-7. 